\title{
Article \\ Combination Constraints of Multiple Fields for Implicit Modeling of Ore Bodies
}

\author{
De-Yun Zhong ${ }^{1}$, Li-Guan Wang ${ }^{1}$ and Jin-Miao Wang ${ }^{1,2, * \mathbb{C}}$ \\ 1 School of Resources and Safety Engineering, Central South University, Changsha 410083, China; \\ deyizhiyun@csu.edu.cn (D.-Y.Z.); liguan_wang@csu.edu.cn (L.-G.W.) \\ 2 School of Environment and Resources, Xiangtan University, Xiangtan 411105, China \\ * Correspondence: Jinmiao_Wang@xtu.edu.cn
}

Citation: Zhong, D.-Y.; Wang, L.-G.; Wang, J.-M. Combination Constraints of Multiple Fields for Implicit Modeling of Ore Bodies. Appl. Sci. 2021, 11, 1321. https://doi.org/ 10.3390/app11031321

Academic Editor: Saro Lee Received: 2 December 2020 Accepted: 27 January 2021 Published: 1 February 2021

Publisher's Note: MDPI stays neutral with regard to jurisdictional claims in published maps and institutional affiliations.

Copyright: (c) 2021 by the authors. Licensee MDPI, Basel, Switzerland. This article is an open access article distributed under the terms and conditions of the Creative Commons Attribution (CC BY) license (https:// creativecommons.org/licenses/by/ $4.0 /)$.
Abstract: In this paper, we introduce combination constraints for modeling ore bodies based on multiple implicit fields interpolation. The basic idea of the method is to define a multi-labeled implicit function that combines different sub-implicit fields by the combination operations, including intersection, union and difference operators. The contribution of this paper resides in the application of combination of more general implicit fields with combination rules for the implicit modeling of ore bodies, such that the geologist can construct constraints honoring geological relationships more flexibly. To improve the efficiency of implicit surface reconstruction, a pruning strategy is used to avoid unnecessary calculations based on the hierarchical bounding box of the operation tree. Different RBF-based methods are utilized to study the implicit modeling cases of ore bodies. The experimental results of several datasets show that the combination constraints are useful to reconstruct implicit surfaces for ore bodies with mineralization rules involving multiple fields.

Keywords: implicit modeling; orebody modeling; radial basis functions; implicit surface; surface reconstruction

\section{Introduction}

Implicit modeling [1-3] of ore bodies consists of two basic processes: the solution of an unknown implicit function and surface reconstruction of the solved implicit function. One of the most significant advantages of implicit modeling is that the topology of the reconstructed surface (the arrangement for how implicit function fields share geometry) is honored automatically when samples are densely distributed. Additionally, the method allows structural geologists to construct additional constraints and update the orebody model dynamically. This has been the research focus in the field of orebody modeling.

Implicit modeling uses an implicit function to represent the orebody model. The distribution of function values is viewed as an implicit function field. Interpolating different attributes (e.g., lithology, grade, distance) of the sampling data produces different implicit function fields. For three-dimensional modeling, the common strategy is to represent the implicit function using a potential field [4] or signed distance field [5]. It tries to find an implicit field function $f(\boldsymbol{x})$ to represent the orebody model. The distance field function divides the mineralized space into three parts according to the function value. Taking the zero-level set as an example, the interior of the orebody isosurface satisfies $f(\boldsymbol{x})<0$, the exterior of the orebody isosurface satisfies $f(\boldsymbol{x})>0$, and the isosurface of orebody satisfies $f(x)=0$. The implicit surface can be obtained by extracting the isosurface with a specified isovalue in the field.

Due to the sparsity of geological sampling data, there is a large uncertainty [6], especially in the topology between drillholes (the arrangement of how sample segments and non-sample segments share geometry) [2], for the interpolation of regions lacking data support. Therefore, it is necessary to append manual constraints according to the geologist's insight. The common implicit functions are often used to interpolate a single 
continuous and smooth model. To extend the construction of interactive constraints, we consider combinations of different types of functions (implicit or explicit), including some unsmoothed functions, to constrain ore bodies. Compared with the single-field method, the multiple-field method can utilize multi-source geology data (e.g., faults, geology interfaces, topography, different mineral assemblages) besides grade data, such that the geologist can construct constraints honoring geological relationships more flexibly. There are many important applications for the combination of multiple fields in orebody modeling. Different types of mineralized, geological (faults, strata, intrusions) and manmade mine engineering features (stopes, shaft, etc.) can be used to model ore bodies by converting the corresponding implicit fields. Another good application is to combine several independent surfaces of orebody to make each part more simply and make them easier to solve. The method can also be used to integrate the modeling results of different implicit modeling methods, and even different modeling methods, to integrate results from explicit and implicit modeling methods.

As an effective geometric representation, implicit representation makes it easy to determine whether a given point is inside or outside an implicit surface by evaluating the corresponding function value. It is very suitable for geometric operations, such as Boolean operations and shape blending, between implicit surfaces. In stratigraphy modeling, geological interfaces can be modeled in chronological order using a combination of multiple potential fields according to pre-defined rules of stratigraphic superposition. The method has been integrated into geological modeling softwares, such as Leapfrog Geo and 3D Geomodeller. Similarly, the idea of a combination of multiple potential fields can be applied to ore-body modeling. This kind of method specifies an implicit field for each implicit function and constructs a combination of constraints for the integration of multiple fields into a single implicit model.

In this paper, we consider applying the constructive solid geometry (CSG) technology to construct combination constraints of multiple fields for the implicit modeling of ore bodies. The basic idea of the method is to define the combined field using a multi-labeled implicit function, and then to construct the combination rules between different sub-fields by the combination of operations or modeling sequences. The contribution of this paper resides in the application of combination of more general implicit fields with combination rules for implicit modeling of ore bodies, which is of interest to the mineral resource modelling community. More generally, the field can be represented by different implicit functions, including some simple geometry functions (the CSG technique), the RBF-based functions [7-9] and the kriging-based functions (the potential field method). In this paper, we try to apply different implicit functions, such as the RBF-based implicit functions and some simple geometry functions, to the combination of multiple fields. In addition, for the implementation of multiple fields, we improve the evaluation efficiency to accelerate the speed of surface reconstruction for the combined field. A pruning strategy is used to avoid unnecessary calculations based on the hierarchical bounding box of the operation tree.

\section{Related Works}

The traditional methods of geostatistics, such as ordinary kriging and simple kriging, are mainly used to construct the block model in grade estimation [10]. Implicit modeling using the universal co-kriging method was developed to model geological interfaces based on the structural geological data in the 2000s, especially the work of Lajaunie et al. [11]. The method, known as the potential field method [12], was developed based on the idea of signed distance field. It is no longer based on the orebody grade interpolation but uses the distance field to compute the implicit function by geological constraints, so that it can be applied to the structural modeling of orebody. To solve the problems of faults and folds in the process of geological modeling, discrete and implicit modeling methods based on scalar fields and volume vector fields are used. For example, Frank et al. [13] used a method based on implicit interpolation and user interaction geological constraints for complex surface reconstruction to solve the fault problem in geological applications. Nicolas et al. [14] 
adopted a scalar field based on sparse data stochastic structure modeling method, to solve the problem of uncertainty and continuity of the fault by combining prior information such as fault direction and the statistical size displacement relationship. Laurent et al. [15] proposed a three-dimensional fault parametric modeling method based on volume vector fields to construct various fault-related displacement models. To solve the geological modeling of folds and fold-superimposed events, Laurent et al. [16] also proposed a method to continuously simulate each fold event by incorporating the constraint of fold geometry into the interpolation method. For the combination of multiple potential fields, Calcagno et al. [4] developed some geological rules to interpolate the geometry domain with multiple geological series. This is a useful method to model objects represented by multiple potential fields. Guillen et al. [17] further considered the geology and the physical properties of rocks using gravity and magnetic data inversion. The Leapfrog software uses the radial basis functions (RBF) method as the implicit function. It is worth noting that the RBF-based methods are the dual form of the kriging-based methods. For example, the generalized radial basis functions (GRBF) method [18-20] is the dual form of the universal co-kriging method. Therefore, the geological rules of the potential field method can be also applied to the RBF-based methods.

The combination rule of potential fields is similar to the CSG technique [21,22] in solid modeling. The basic idea is to construct a complex model to combine simpler models by using Boolean operations on sets, including union, intersection and difference. Wyvill et al. $[23,24]$ extended the CSG tree and called the structure of arbitrary compositions of models the BlobTree. The Boolean operations, as well as blending and warping operations, were viewed as nodes in the BlobTree. Based on the blending advantage $[25,26]$ of implicit functions, many composition operators that have been proposed to construct complex implicit models. To improve the convenience of blending manipulation, Barthe et al. [27] used a free-form, curve-controlled point-by-point to define the blending range between implicit models. Li [28] proposed a set of smooth piecewise polynomial blending operations to control the smoothness of the blending range for implicitly defined geometric shapes. It is worth noting that the combination constraints can be applied not only to implicit representation and parametric representation [29], but also to explicit representation. Based on the idea of a signed distance field, it is natural to convert explicit representations into implicit representations to restrict the orebody model. For example, Zhan et al. [30] proposed an interactive method to clip the implicit model using the blending operations of a series of plane widgets. Recently, Collon et al. [1] studied the combination constraints of implicit-surface- and Voronoi-based remeshing meshes.

\section{Implicit Modeling}

To recover an arbitrarily complex orebody model, a set of basis functions should be found in a certain function space (e.g., radial basis functions space) to approximate the unknown continuous implicit function $f(x)$ representing the orebody model. Considering that different implicit functions have their own advantages and disadvantages, it is necessary to choose a method that can construct a variety of interpolation constraints.

In this paper, we focus on the RBF-based interpolation functions $s(x)$ for their capability of extraordinary extrapolation in sparse data environment, including the RBF interpolant, the HRBF interpolant [31] and the GRBF interpolant [20]. The RBF-based methods can be used to construct geology constraints based on three basic interpolation constraints, including the domain constraint, the gradient constraint and the tangent constraint. Among them, the domain constraint is used to control the domain value (function value) at a given point. The traditional RBF method can only construct the domain constraint, the HRBF method adds the gradient constraint, and the GRBF method adds the tangent constraint, as shown in Table 1. 
Table 1. The RBF-based interpolation methods.

\begin{tabular}{cc}
\hline Interpolant & Constraints \\
\hline RBF & domain constraints \\
HRBF & domain constraints, gradient constraints \\
GRBF & domain constraints, gradient constraints, tangent constraints \\
\hline
\end{tabular}

(1) RBF

The RBF interpolant has the form

$$
s(\boldsymbol{x})=\sum_{j=1}^{\mu} a_{j} \Phi\left(\boldsymbol{x}, \boldsymbol{x}_{j}\right)+p(\boldsymbol{x})
$$

where $\mu$ is the number of domain constraints, $a_{j}$ and polynomial coefficients are to be determined, $\Phi\left(\boldsymbol{x}, \boldsymbol{x}_{j}\right)$ is a usual radial basis function and $p(\boldsymbol{x})$ is a low-degree polynomial.

(2) HRBF

The HRBF interpolant has the form

$$
s(\boldsymbol{x})=\sum_{j=1}^{\mu} a_{j} \Phi\left(\boldsymbol{x}, \boldsymbol{x}_{j}\right)+\sum_{k=1}^{\sigma}\left\langle\boldsymbol{b}_{k}, \nabla^{\prime} \Phi\left(\boldsymbol{x}, \boldsymbol{x}_{\mu+k}\right)\right\rangle+p(\boldsymbol{x})
$$

where $\sigma$ is the number of gradient constraints, $a_{j}$ are scalar coefficients, $\boldsymbol{b}_{k}$ are vector coefficients with three components, $\nabla^{\prime}$ is the differential operator with respect to the second variable of $\Phi\left(x, x_{\mu+k}\right)$ and $\langle.,$.$\rangle is an inner product operation of two vectors.$

(3) GRBF

The GRBF interpolant has the form

$s(\boldsymbol{x})=\sum_{j=1}^{\mu} a_{j} \Phi\left(\boldsymbol{x}, \boldsymbol{x}_{j}\right)+\sum_{k=1}^{\sigma}\left\langle\boldsymbol{b}_{k}, \nabla^{\prime} \Phi\left(\boldsymbol{x}, \boldsymbol{x}_{\mu+k}\right)\right\rangle+\sum_{l=1}^{\tau} e_{l}\left\langle\boldsymbol{t}_{\mu+\sigma+l}, \nabla^{\prime} \Phi\left(\boldsymbol{x}, \boldsymbol{x}_{\mu+\sigma+l}\right)\right\rangle+p(\boldsymbol{x})$

where $\tau$ is the number of tangent constraints, $a_{j}, e_{l}$ are scalar coefficients and $\boldsymbol{b}_{k}$ are vector coefficients with three components.

To obtain the interpolation function $s(\boldsymbol{x})$ representing the orebody model, it is necessary to solve the linear system composed of interpolation constraints.

(1) Domain constraint

The domain constraint satisfies

$$
f\left(x_{i}\right)=f_{i}, \quad i=1,2, \ldots, \mu
$$

where $f_{i}$ is the function value at $x_{i}$.

(2) Gradient constraint

The gradient constraint satisfies

$$
\nabla f\left(x_{i}\right)=n_{i}, \quad i=\mu+1, \mu+2, \ldots, \mu+\sigma
$$

where $\boldsymbol{n}_{i}$ is the gradient vector at $\boldsymbol{x}_{i}$.

(3) Tangent constraint

The tangent constraint satisfies

$$
\left\langle\nabla f\left(\boldsymbol{x}_{i}\right), \boldsymbol{t}_{i}\right\rangle=0, \quad i=\mu+\sigma+1, \mu+\sigma+2, \ldots, \mu+\sigma+\tau
$$

where $\boldsymbol{t}_{i}$ is the tangent vector at $x_{i}$.

To ensure that the interpolation function $s(x)$ can recover all model features of the unknown implicit function $f(\boldsymbol{x})$, it must be ensured that the radial basis functions space is well-posed (sampling is sufficient). 
Note that different interpolation constraints yield different linear systems for the interpolation method. The corresponding solution and surface reconstruction methods are also different. A good strategy is to automatically select the appropriate solution and surface reconstruction methods according to the type of interpolation constraints. The combination constraints are independent of the implicit function used. Therefore, each field can be interpolated by different RBF-based methods. It is possible to use different implicit functions representing the orebody model for each sub-field of the combined field. For example, it is better to use the GRBF method with strong constraints in a sparse data environment and the RBF method with efficient performance in a dense data environment. The type of constraints used in a sub-field determines the corresponding RBF-based interpolation method.

\section{Combination Constraints}

\subsection{Multiple Fields}

\subsubsection{Multi-Labeled Implicit Function}

For the signed distance field, the function values of $f(x)$ are represented as signed distances. The function value of any point $p$ in the mineralized space is defined as the distance from $p$ to the closest point on the implicit surface $S$. The points on the surface satisfy $S=\left\{x \in R^{3} \mid f(x)=0\right\}$. The interior and exterior of the surface can be distinguished based on the sign of function values. Without loss of generality, we make the agreement that the interior of the surface is negative, and the exterior of the surface is positive, as shown in Figure 1.

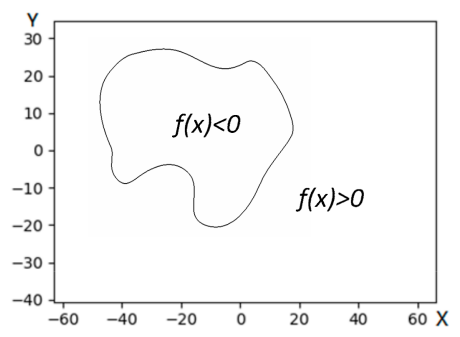

(a)

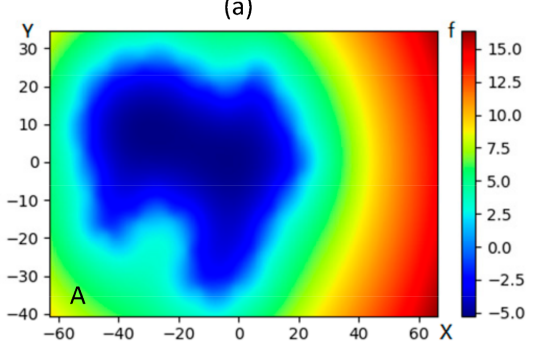

(c)

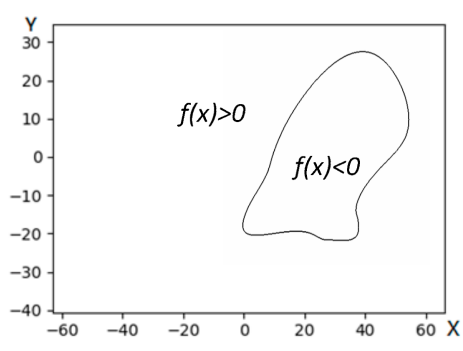

(b)

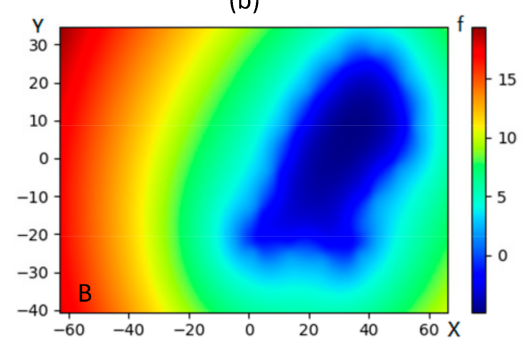

(d)

Figure 1. Schematic diagram of two signed distance fields $(\mathbf{c}, \mathbf{d})$ interpolated from two contours $(\mathbf{a}, \mathbf{b})$. The range of signed distance values corresponds to the color bar.

For the modeling space involving several fields, the existing multi-labeled implicit function $[32,33]$ can be used to define the combined field. In this definition, each sub-field is represented by a labeled implicit function. The function value of a point in each sub-field is also assigned a corresponding label, and the valued function of a point in the combined field is determined by the multi-labeled values according to the combination rules.

The multi-labeled implicit function $F(\boldsymbol{x})$ of the combined field can be defined as

$$
F(x)=f_{1}(x) \circ f_{2}(x) \circ \ldots \circ f_{n}(x)
$$

where $f_{i}(\boldsymbol{x})$ is the implicit function of the $i$-th sub-field and the symbol $\circ$ indicates a certain combination rule between fields. 
The combined field is still represented by an implicit function. The modeling goal is to reconstruct the combined implicit function $F(x)$. Compared with the single-field function, the main difference is that the function value of $F(x)$ should be determined by several subfunctions via combination rules. As mentioned, each sub-field of the combined potential field can be expressed by different implicit functions. For example, $f_{i}(\boldsymbol{x}), i=1,2, \ldots, n$ can be interpolated by different RBF-based methods. Therefore, the appropriate interpolation constraints and the corresponding implicit modeling method can be selected as needed for each sub-field.

\subsubsection{Bounding Box}

To visualize an implicit function, the surface reconstruction method (e.g., the marching cubes method [34]) is used to extract isosurface by polygonizing a specified bounding box (e.g., a rectangle bounding box). As the function values outside the bounding box will not be evaluated, the bounding box can be also used to limit the interaction range of the implicit function field.

For the multi-labeled implicit function, we can specify a rectangle bounding box based on the corresponding sampling data for each sub-field. The implicit field of bounding box can be expressed by a signed distance function using the distance computation from a target point to the surface $S$ of bounding box. The bounding box of the $i$-th sub-field can be defined as

$$
B_{f_{i}}(\boldsymbol{x})= \pm \operatorname{Dist}(\boldsymbol{x}, \boldsymbol{S})
$$

where $\operatorname{Dist}(\boldsymbol{x}, \boldsymbol{S})$ is the minimum distance from $x$ to $S$. The sign of $B_{f_{i}}(x)$ indicates whether the evaluation point is inside or outside the bounding box. The range of the sub-field is limited to the interior of the bounding box. Therefore, the $i$-th labeled implicit function $f_{i}(\boldsymbol{x})$ works only if $B_{f_{i}}(\boldsymbol{x}) \leq 0$. Besides limiting the range of sub-fields, the bounding box is useful to accelerate the evaluation speed of function values for the combined implicit function $F(\boldsymbol{x})$.

The minimum bounding box of a field can be automatically calculated by the sampling data and scaled by a certain scale factor. There are two different types of bounding boxes, axis-aligned bounding box (AABB) and oriented bounding box (OBB) [35]. Compared with the axis-aligned bounding box, the oriented bounding box is beneficial to further improve the efficiency of evaluating the implicit function $F(x)$. The oriented bounding box follows the shape of the ore body well, especially for the thin ore bodies. It reduces the number of locations where the implicit function in surface reconstruction is evaluated. For the multilabeled implicit function, it is good to avoid unnecessary calculations of some sub-fields in certain locations. We will discuss the evaluation efficiency in the Implementation section.

The optimal oriented bounding box can be calculated by the principal component analysis (PCA) method [36,37]. The basic idea of the PCA method is to construct a $3 \times 3$ covariance matrix $C$ by using the coordinate differences of the sampling points with respect to the centroid in the direction of three coordinate axes. The eigenvectors of the covariance matrix are the three main directions of the optimal oriented bounding box. Given a set of $N$ sampling points $\left\{\boldsymbol{p}_{k} \mid \boldsymbol{p}_{k}=\left(p_{k, 1}, p_{k, 2}, p_{k, 3}\right), k=1,2, \ldots, N\right\}$, the ij-th component of the corresponding covariance matrix $C$ can be computed as

$$
C_{i j}=\frac{1}{N} \sum_{k=1}^{N}\left(p_{k, i}-c_{i}\right)\left(p_{k, j}-c_{j}\right), 1 \leq i, j \leq 3
$$

where $c=\left(c_{1}, c_{2}, c_{3}\right)$ is the centroid of the sampling points.

\subsection{Combination Rules}

Combination rules are used to determine the function value of the multi-labeled implicit function $F(x)$ based on the combination operations or modeling sequence of the sub-fields. 


\subsubsection{Combination Operations}

Similar to the Boolean operations of the CSG technology, three basic combination operations of intersection operation, union operation and difference operation are defined. The set operations are calculated with respect to the interior of the sub-fields. For a given point, as long as the sign of the function value of the point is evaluated, the position of the point in each sub-field is easy to determine. Taking the two intersected fields A and B in Figure 1 as an example, Figure 2 shows the signed distance values of the combined field using a different combination of operations.

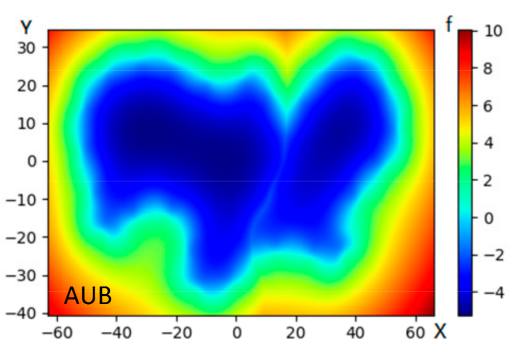

(a)

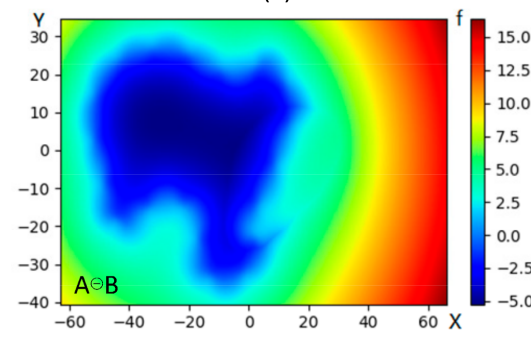

(c)

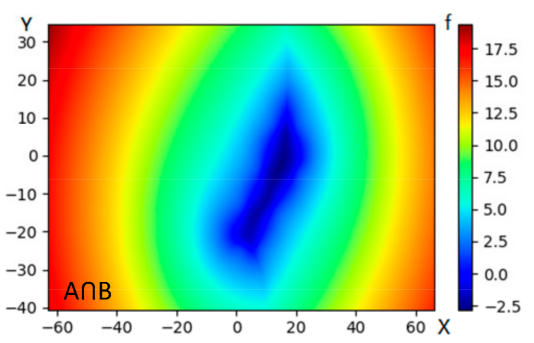

(b)

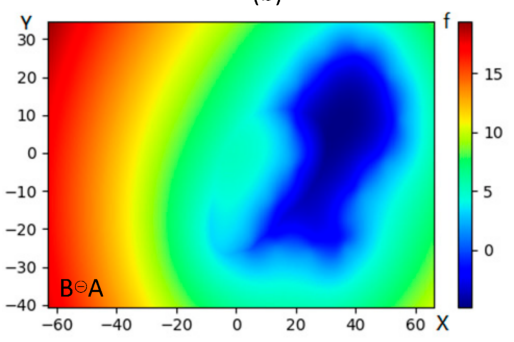

(d)

Figure 2. Combination operations of two fields A and B: (a) union operation $A \cup B$; (b) intersection operation $A \cap B ;(\mathbf{c})$ difference operation $A \ominus B ;(\mathbf{d})$ difference operation $B \ominus A$. The range of signed distance values corresponds to the color bar.

(1) Intersection operation

The intersection operation of two fields $\mathrm{A}$ and $\mathrm{B}$, denoted as $A \cap B$, is the combined field containing the intersected interior of $A$ and $B$. The combined field of $A \cap B$ is expressed as

$$
F(\boldsymbol{x})=f_{A \cap B}(\boldsymbol{x})=\max \left(f_{A}(\boldsymbol{x}), f_{B}(\boldsymbol{x})\right)
$$

where $f_{A}(x)$ is the implicit function of field $A$ and $f_{B}(x)$ is the implicit function of field $B$.

The combined bounding box of the combined field, denoted as $B_{F}(x)$, is expressed as

$$
B_{F}(\boldsymbol{x})=B_{f_{A \cap B}}(\boldsymbol{x})=\max \left(B_{f_{A}}(\boldsymbol{x}), B_{f_{B}}(\boldsymbol{x})\right)
$$

where $B_{f_{A}}(\boldsymbol{x})$ is the implicit function of the bounding box with respect to field $A$ and $B_{f_{B}}(\boldsymbol{x})$ is the implicit function of the bounding box with respect to field $B$.

(2) Union operation

The union operation of two fields $A$ and $B$, denoted as $A \cup B$, is the combined field containing both the interior of $A$ and $B$. The combined field of $A \cup B$ is expressed as

$$
F(\boldsymbol{x})=f_{A \cup B}(\boldsymbol{x})=\min \left(f_{A}(\boldsymbol{x}), f_{B}(\boldsymbol{x})\right)
$$

The combined bounding box of the combined field is expressed as

$$
B_{F}(\boldsymbol{x})=B_{f_{A \cup B}}(\boldsymbol{x})=\min \left(B_{f_{A}}(\boldsymbol{x}), B_{f_{B}}(\boldsymbol{x})\right)
$$




\section{(3) Difference operation}

The difference operation of two fields $A$ and $B$, denoted as $A \odot B$, is the combined field containing the intersected interior of $A$ and $-B .-B$ is the complement operation of $B$. The complement operation satisfies $f_{-B}(x)=-f_{B}(x)$. The combined field of $A \odot B$ is expressed as

$$
F(\boldsymbol{x})=f_{A \ominus B}(\boldsymbol{x})=f_{A \cap(-B)}(x)=\max \left(f_{A}(\boldsymbol{x}),-f_{B}(\boldsymbol{x})\right)
$$

The combined bounding box of the combined field is expressed as

$$
F(x)=f_{A \ominus B}(x)=f_{A \cap(-B)}(x)=\max \left(f_{A}(x),-f_{B}(x)\right)
$$

In the practice of orebody modeling, some complex combination rules can be combined based on the above combination operations. The hierarchical combination operations lead to an operation tree. The intersection operation, union operation and difference operation are viewed as nodes in the operation tree. A leaf node of the operation tree corresponds to a sub-field, a non-leaf node corresponds to a combined sub-field, and the root node corresponds to the final combined field.

\subsubsection{Modeling Sequence}

If it is easy to determine the priorities of the overlapping fields (e.g., the geological surfaces with chronological order), the modeling sequence of sub-fields is more convenient than combination operations to construct combination rules. In this case, each sub-field is specified as a priority. The multi-labeled function value of the combined field can be determined simply, based on the priority of the sub-fields. For a point with multiple intersected sub-fields, its combined value is equal to the labeled value with the highest priority. Consequently, a sub-field with higher priority allows for truncating and cutting across the lower ones. On the contrary, a sub-field with lower priority cannot change the geometry shape of the higher ones.

The modeling sequence of multiple fields can be also determined by the combination operations between sub-fields. Taking the two intersected fields A and B in Figure 1 as an example, if the priority of field $\mathrm{A}$ is higher than field $\mathrm{B}$, the multi-labeled implicit function $F(x)$ of the combined field with respect to field B is $F(x)=f_{B}(x) \ominus f_{A}(x)$. In stratigraphy modeling, the chronological order of series can be determined by the modeling sequence. A stratigraphic pile [4] is defined to specify the modeling sequence. Besides the time-order of series, two basic combination rules should be specified to determine how a series impacts on a model, Onlap and Erode relations [4], which can be combined to define complex geological rules.

\subsection{Combined Representations}

Besides the implicit representation, combination constraints can be also used to blend the modeling results of explicit representation and parametric representation.

The basic idea of combined representations is that the modeling result can be converted as an implicit function field. For example, the modeling result of explicit modeling can be converted into a signed distance field by computing the minimum distance between an evaluating point and the nearest point on the mesh. The distance is signed according to the mesh normal direction. For a closed surface, the region pointed by the positive direction of the mesh normal is outside the surface (the sign of the function value is positive), and the region pointed by the negative direction of the mesh normal is inside the surface (the sign of the function value is negative). Different modeling methods have their own advantages and disadvantages. For the modeling results using different modeling methods, it is necessary to blend the reconstructed meshes into the implicit representation of orebody.

Based on the idea of a signed distance field, some geometry functions composed of simple geometry equations can be used to construct signed distance functions naturally. Compared with the interpolation constraints, the geometry constraints can be used to limit or extend the interpolation trend of orebody model more efficiently. 
(1) Plane constraint

The signed distance from a point to a plane can be represented as a simple implicit function

$$
f(\boldsymbol{x})=\frac{n_{x}\left(x-x_{0}\right)+n_{y}\left(y-y_{0}\right)+n_{z}\left(z-z_{0}\right)}{\sqrt{n_{x}^{2}+n_{y}^{2}+n_{z}^{2}}}
$$

where $x=(x, y, z)$ is an evaluation point in the distance field, $x_{0}=\left(x_{0}, y_{0}, z_{0}\right)$ is a point on the plane and $\boldsymbol{n}=\left(n_{x}, n_{y}, n_{z}\right)$ is the normal vector of the plane. The sign of $f(\boldsymbol{x})$ indicates whether the evaluation point is inside or outside the model. The bounding box is used to define the extent of the plane. Clearly, the region pointed to by the positive direction of the normal is exterior, and the region pointed to by the negative direction of the normal is interior.

(2) Line constraint

To distinguish the exterior and interior of a distance field constructed by a line, define a plane passing through a given line as a projection plane. Construct a $2 \mathrm{D}$ coordinate system on a projection plane. Then, the signed projection distance from a point to the line in a specified projection plane can be represented as a simple implicit function

$$
f(\boldsymbol{x})=\frac{n_{x}\left(x^{\prime}-x_{0}\right)+n_{y}\left(y^{\prime}-y_{0}\right)}{\sqrt{n_{x}^{2}+n_{y}^{2}}}
$$

where $x^{\prime}=\left(x^{\prime}, y^{\prime}\right)$ is the $2 \mathrm{D}$ projection coordinate of the projection plane with respect to the evaluation point $\boldsymbol{x}=(x, y, z), \boldsymbol{x}_{0}=\left(x_{0}, y_{0}, z_{0}\right)$ is a point on the line and $\boldsymbol{n}=\left(n_{x}, n_{y}\right)$ is the $2 \mathrm{D}$ normal vector of the line on the projection plane. The bounding box is used to define the extent of the line.

(3) Surface constraint

Some common primitive objects can be also used to construct signed distance functions. Taking the sphere as an example, the signed distance from a point to the surface of a sphere can be represented as a simple implicit function

$$
f(x)=\sqrt{\left(x-x_{0}\right)^{2}+\left(y-y_{0}\right)^{2}+\left(z-z_{0}\right)^{2}}-R
$$

where $x=(x, y, z)$ is an evaluation point in the distance field, $x_{0}=\left(x_{0}, y_{0}, z_{0}\right)$ is the center of the sphere and $R$ is the radius of the sphere.

\section{Implementation}

The implementation of implicit modeling includes three main steps: evaluation, solution, and surface reconstruction. The evaluation step of implicit function is the basis of the solution and surface reconstruction steps. The efficiency of iterative solution and isosurface extraction depends on the efficiency of evaluation. Each sub-field of the combined implicit function field can be solved separately. The implicit surface with multiple fields should be reconstructed using multi-domain (or multi-material) isosurface extraction methods [34,38]. If the combined potential field implicit function is regarded as a single field, the surface reconstruction of the combined potential field is the same as the single field.

We mainly focus on the evaluation of the combined implicit function. For a given point, there are several labeled function values to be determined for each sub-field, which reduces the efficiency of evaluation. In fact, it is not necessary to calculate some invalid labeled function values for the points outside the corresponding bounding box. For the combination rules with modeling sequence, the sub-fields can be evaluated in order of priority, from high to low. Moreover, compared with the evaluation of function value, it is more efficient to evaluate whether a point is inside a bounding box or not. Therefore, more than one sub-field is evaluated only in the region where the corresponding bounding boxes overlap. 
Every node of the operation tree is specified a bounding box. The function values can be evaluated in a traversal way based on the hierarchical bounding box of the operation tree. For a given point, some invalid nodes will not be evaluated to avoid unnecessary calculations. Based on the above analysis, the evaluation efficiency of the combined implicit function is improved to accelerate the speed of implicit surface reconstruction.

Given a point $x$, the improved evaluation method for the combined implicit function $F(x)$ is given below.

Step 1: Solve the implicit function for each sub-field separately;

Step 2: Obtain the operation tree according to the specified hierarchical combination operations;

Step 3: Specify the bounding box for each leaf node of the operation tree. For a leaf node, compute the minimum bounding box based on the corresponding sampling data. Then, extend the box by a factor according to the reconstruction result until it obtains a desired result;

Step 4: Traversing the operation tree from bottom to top, compute the optimal oriented bounding box for each non-leaf node of the operation tree. Among them, the bounding box of a non-leaf node is calculated by the combination operation of its child nodes;

Step 5: Traversing the operation tree from top to bottom, evaluate whether the given point $x$ is inside the bounding box of each node or not. If $x$ is inside the bounding box of a node $M_{i}$, node $M_{i}$ is a valid node and its child nodes should be judged in the same way. If $x$ is outside the bounding box of a node $N_{i}$, node $N_{i}$ and all of its child nodes are invalid nodes;

Step 6: After the traversal is completed, the valid sub-fields $\left\{f_{i}\right\}$ in the leaf nodes that have an influence on the evaluation point $x$ can be obtained;

Step 7: Compute the corresponding labeled function values $\left\{f_{i}(\boldsymbol{x})\right\}$ of the sub-fields at the valid leaf nodes. For the RBF-based implicit functions, the fast multipole method [39] can be used to further improve the evaluation efficiency of sub-fields;

Step 8: Traversing the operation tree from bottom to top, compute the function value of each node that has an influence on the evaluation point $x$ according to the corresponding combination operations;

Step 9: Finally, the function value of the root node is the combined value of $F(x)$.

\section{Case Studies}

Different combination rules yield different modeling results. The appropriate combination operations or modeling sequence should be specified according to the corresponding geological rule. The RBF-based methods were implemented to interpolate the raw drillhole data [40] and the user-defined contour data [41] for each sub-field. Several examples of orebody implicit modeling were studied to analyze the combination constraints of multiple fields.

The combination constraints can be used to restrict the implicit surface based on the modeling result of explicit modeling. For example, the signed distance field converted from an explicit mesh can be used to restrict the implicit surface of orebody based on specific distance values. As shown in Figure 3b, without the constraint of topography, the interpolated model (implicit mesh generated by implicit method) may extend above the topography surface (explicit mesh generated by explicit method). A combination constraint is used to ensure an exact fit between the top of the ore body and topography in Figure 3c. The corresponding multi-labeled implicit function $F(\boldsymbol{x})$ is $F(\boldsymbol{x})=f_{2}(\boldsymbol{x}) \ominus f_{1}(\boldsymbol{x})$, where $f_{1}(\boldsymbol{x})$ represents the topography and $f_{2}(x)$ represents the orebody. Another combination constraint is used to constrain the interpolated model to $45 \mathrm{~m}$ below the topography in Figure $3 \mathrm{~d}$. In this case, the corresponding multi-labeled implicit function $F(x)$ is $F(x)=f_{2}(x) \ominus\left(f_{1}(x)-45\right)$. It is worth noting that the region above the topography is viewed as the interior of the model, though the topography surface is not closed. 


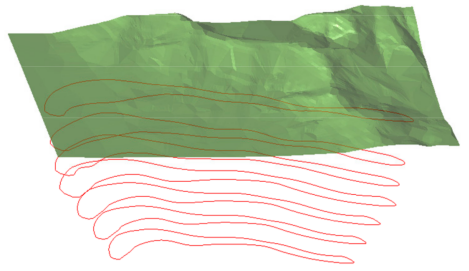

(a)

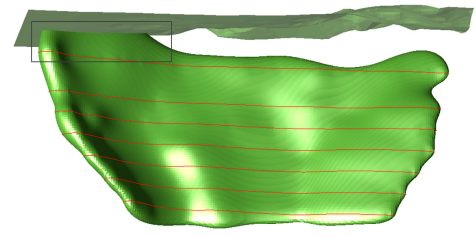

(c)

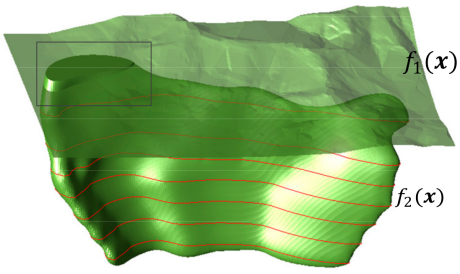

(b)

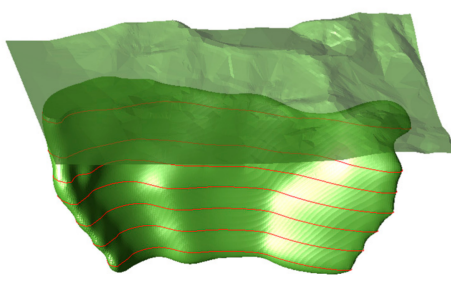

(d)

Figure 3. Combination constraint of the topography surface based on the signed distance field: (a) the topography surface and interpreted contours; (b) the interpolated model without the constraint of topography; (c) the interpolated model with a combination constraint; (d) the interpolated model with another combination constraint.

To make full use of the sampling data of production exploration, the orebody can be modeled in blocks at different stages of mine production, and the modeling results between blocks are kept independent of each other. The three blocks are clipped by different overlapping sub-fields, respectively, to ensure the continuity at the connection of regions. A block corresponds to a sub-field. The blocks are blended using combination operations. If the additional sampling data are concentrated in a new block, the modeling result will not affect the old blocks in the updating process of orebody. Figure 4 demonstrates an example of block modeling with three blocks modeled at different stages. The multi-labeled implicit function $F(\boldsymbol{x})$ of this example is $F(\boldsymbol{x})=f_{1}(\boldsymbol{x}) \cup f_{2}(\boldsymbol{x}) \cup f_{3}(\boldsymbol{x})$, where $f_{1}(\boldsymbol{x})$ represents the upper block, $f_{2}(x)$ represents the middle block and $f_{3}(x)$ represents the lower block.

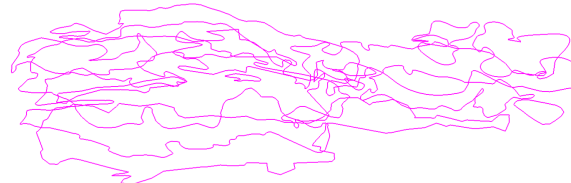

(a)

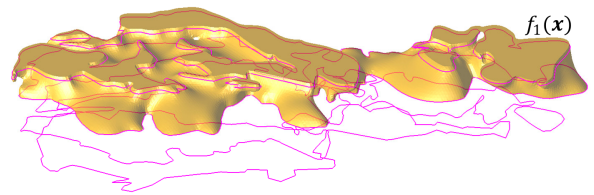

(c)

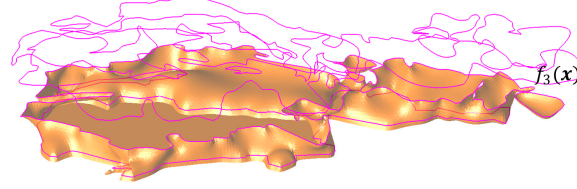

(e)

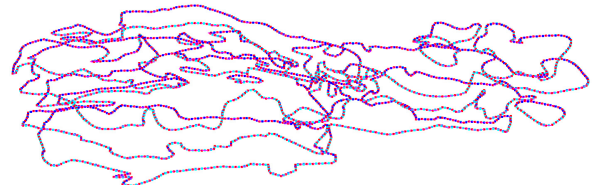

(b)

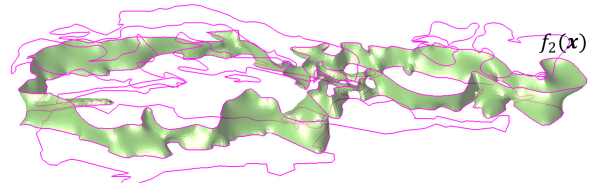

(d)

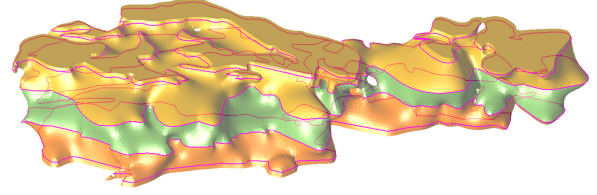

(f)

Figure 4. Combination constraint of the block modeling at different stages: (a) the interpreted contours; (b) the interpolation constraints sampled from the contours; (c-e) are the modeling results at different stages; (f) the combined result. 
Implicit function fields are generally represented by continuous implicit functions. However, the mineralization space with faults is divided into several discontinuous regions. To consider the influence of faults, the combination constraints can be used to divide the discontinuous mineralization space into several continuous subspaces for interpolation. The interface of fault is interpolated as an independent sub-field, and the interpolation constraints are separated by the interface of fault. As shown in Figure 5, there are three sub-fields, including the fault and two regions divided by the fault. The multi-labeled implicit function $F(x)$ of the hanging wall orebody is $F(x)=f_{1}(x) \ominus f_{3}(x)$. The multilabeled implicit function $F(x)$ of the foot wall orebody is $F(x)=f_{2}(x) \ominus\left(-f_{3}(x)\right)$, where $f_{1}(\boldsymbol{x})$ represents the hanging wall orebody, $f_{2}(\boldsymbol{x})$ represents the foot wall orebody and $f_{3}(\boldsymbol{x})$ represents the fault. The foot wall of the fault is viewed as the interior of the model though the fault surface is not closed.

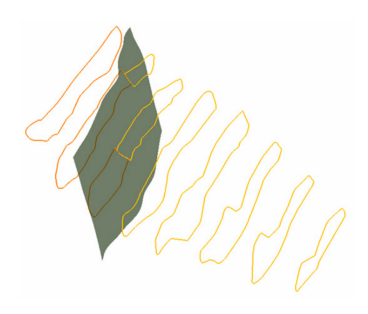

(a)

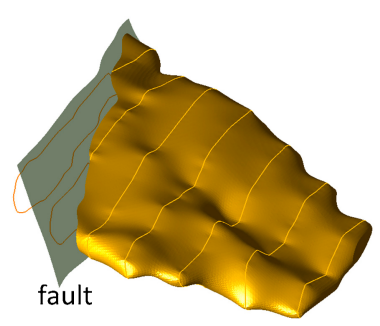

(c)

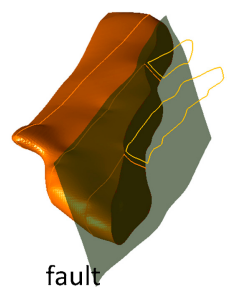

(b)

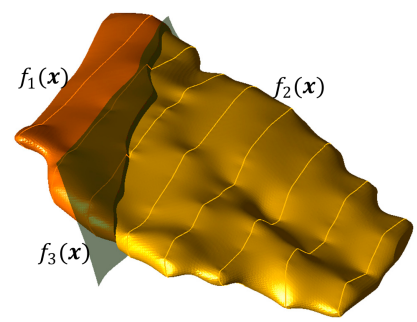

(d)

Figure 5. Combination constraint of the discontinuous mineralization space with a fault: (a) the fault surface and interpreted contours; (b) the combined hanging wall orebody; (c) the combined foot wall orebody; (d) the combined results.

The combination constraints are useful to recover the distribution of mineralization trend according to the mineralization rules involving several fields. For the stratabound ore deposit, the interpolation constraints that are only converted from the grade data are difficult to use to recover the grade distribution according to the mineralization rule. The multiple fields can be used to represent the relationship between orebody and geological formations. Figure 6 demonstrates a stratabound orebody that contacts above a geological interface. The multi-labeled implicit function $F(x)$ of this example is $F(\boldsymbol{x})=f_{1}(\boldsymbol{x}) \cap\left(f_{2}(\boldsymbol{x}) \cap f_{4}(\boldsymbol{x})\right)$, where $f_{1}(\boldsymbol{x})$ is the line constraints used to restrict the model boundary, $f_{2}(x)$ represents the hanging side surface of the thin orebody, $f_{3}(x)$ represents the foot side surface of the thin orebody and $f_{4}(x)$ represents the geological interface. Without the constraint of geological interface, the multi-labeled implicit function $F(x)$ is $F(\boldsymbol{x})=f_{1}(\boldsymbol{x}) \cap\left(f_{2}(\boldsymbol{x}) \cap f_{3}(\boldsymbol{x})\right)$. The thin orebody is modeled by two separated sub-fields and clipped by several line constraints. 


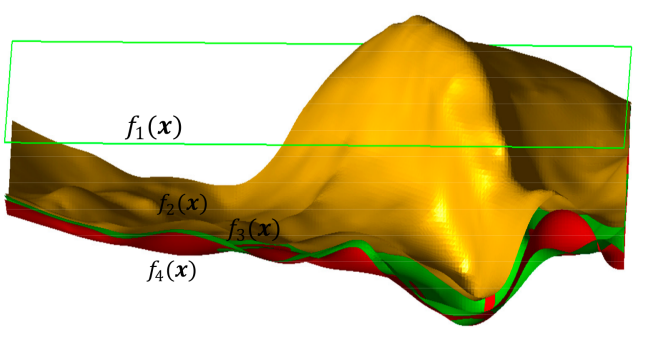

(a)

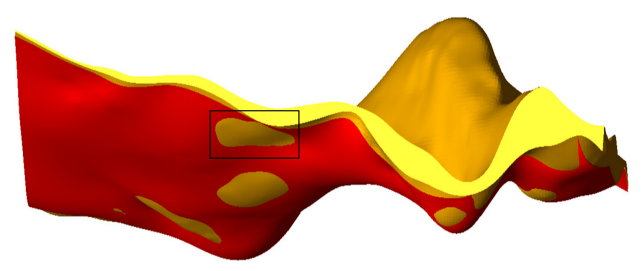

(c)

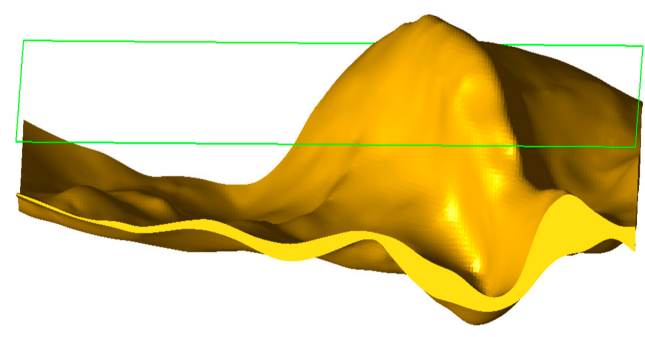

(b)

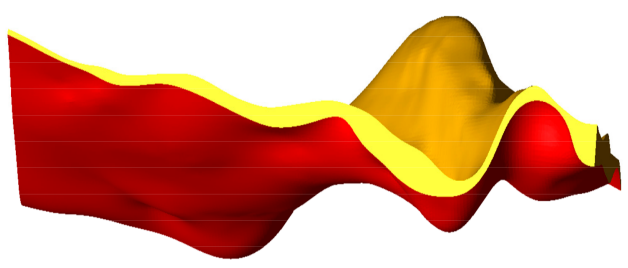

(d)

Figure 6. Combination constraint of the stratabound ore deposit: (a) the hanging side surface of orebody (yellow), the foot side surface of orebody (green), the geological interface (red) and the line constraints; $(\mathbf{b}, \mathbf{c})$ show the interpolated model without considering the geological interface; (d) the interpolated model with the constraint of the geological interface.

\section{Discussion and Conclusions}

In this paper, we introduce combination constraints of multiple fields for the implicit modeling of ore bodies based on the idea of implicit function field interpolation. Three basic combinations of intersection, union and difference are defined to represent the combined relationship between sub-fields. The combined field is constructed according to the definition of multi-labeled implicit functions and combination rules. The method can be also used to blend the modeling results of explicit representation and parametric representation. To accelerate the speed of implicit surface reconstruction, the evaluation efficiency of the combined implicit function is improved based on the hierarchical structure of the operation tree. The RBF-based methods are applied to the modeling problem of drillhole data interpolating and contour data interpolating. The experimental results of several datasets demonstrate that the combination constraints of multiple fields apply to a range of typical situations in the implicit modeling of orebody.

In the practice of orebody modeling, it is sometimes difficult to recover complex orebody models using a modeling method. Combination constraints of multiple fields are useful for integrating the modeling results of different implicit modeling methods (including the kriging-based methods). Each sub-field can be interpolated by an appropriate RBF-based method according to the type of constraints. Therefore, the RBF-based methods are combined to facilitate the application of multiple implicit modeling methods. Furthermore, some explicit functions can be converted into implicit functions based on the idea of signed distance field. Several geometry constraints are constructed to control the extrapolation of orebody model. As the examples demonstrated, combination constraints can be used to reconstruct the orebody model based on mineralization rules involving several fields.

There are several limitations that need to be improved. Although four different cases of combination operations for multiple fields are exemplified, due to the changeable geological conditions, it is necessary to further study the combination constraints to conveniently honor the geological rules involving several fields. The combination constraints require that the different subfields are independent. For example, aiming at the modeling of the orebody where the mineralization space with faults is divided into several discontinuous regions, this paper only uses combination constraints to divide the discontinuous mineralization space into several continuous subspaces for interpolation. However, when the 
mineralization space is transferred due to geological events, the corresponding fields are not independent. Therefore, it is necessary to further study the shape blending of multiple fields under various geological conditions. Additionally, to subdivide the complex surface into simple operations, the continuity of adjacent blocks should be further studied for block modeling.

Implicit modeling is a promising method for orebody modeling. However, for sparse drillhole data with large intervals, the reconstructions without manual constraints are likely to produce discontinuities. The reliability of implicit modeling from the sparse drillhole data depends, to a large extent, on the construction of the interpolation constraints and geological rules. Therefore, the modeling method still requires the knowledge of a structural geologist's insight and this input is made in the form of combination constraints, additional sections, structural anisotropy and other geological rules. More flexible constraints should be studied in future works to avoid undesirable artifacts.

Author Contributions: D.-Y.Z. and L.-G.W. conceived, designed, and performed the experiments; J.M.W. analyzed the data and revised the methodology; D.-Y.Z. wrote the paper; all authors discussed the results and revised the paper. All authors have read and agreed to the published version of the manuscript.

Funding: This work was financially supported by the National Key R\&D Program of China (2017YFC0602905) and the Key R\&D Program of Hunan Province, China (2018SK2053).

Institutional Review Board Statement: Not applicable.

Informed Consent Statement: Not applicable.

Data Availability Statement: Not applicable.

Acknowledgments: Thanks to the public datasets used in this research. We also thank the reviewers for their comments and suggestions to improve the quality of the paper.

Conflicts of Interest: The authors declare no conflict of interest. The funders had no role in the design of the study; in the collection, analyses, or interpretation of data; in the writing of the manuscript, and in the decision to publish the results.

\section{References}

1. Collon, P.; Steckiewicz-Laurent, W.; Pellerin, J.; Laurent, G.; Caumon, G.; Reichart, G.; Vaute, L. 3d geomodelling combining implicit surfaces and voronoi-based remeshing: A case study in the lorraine coal basin (france). Comput. Geosci. 2015, 77, 29-43. [CrossRef]

2. Miguel, D.L.V.; Wellmann, J.F. Structural geologic modeling as an inference problem: A Bayesian perspective. Interpretation 2016, 4, SM1-SM16.

3. Cowan, E.; Beatson, R.; Fright, W.; McLennan, T.; Mitchell, T. Rapid geological modelling. In Proceedings of the Applied Structural Geology for Mineral Exploration and Mining International Symposium, Kalgoorlie, Australia, 23-25 September 2002.

4. Calcagno, P.; Chilès, J.-P.; Courrioux, G.; Guillen, A. Geological modelling from field data and geological knowledge: Part i. Modelling method coupling 3d potential-field interpolation and geological rules. Phys. Earth Planet. Inter. 2008, 171, 147-157. [CrossRef]

5. Hjelle, Ø.; Petersen, S.A.; Bruaset, A.M. A numerical framework for modeling folds in structural geology. Math. Geosci. 2013, 45, 255-276. [CrossRef]

6. Dimitrakopoulos, R. Conditional simulation algorithms for modelling orebody uncertainty in open pit optimisation. Int. J. Surf. Min. Reclam. Environ. 1998, 12, 173-179. [CrossRef]

7. Majdisova, Z.; Skala, V. Radial basis function approximations: Comparison and applications. Appl. Math. Model. 2017, 51, 728-743. [CrossRef]

8. Carr, J.C.; Beatson, R.K.; Cherrie, J.B.; Mitchell, T.J.; Fright, W.R.; McCallum, B.C.; Evans, T.R. Reconstruction and representation of 3D objects with radial basis functions. In Proceedings of the 28th Annual Conference on Computer Graphics and Interactive Techniques, Los Angeles, CA, USA, 12-17 August 2001; pp. 67-76.

9. Cuomo, S.; Galletti, A.; Giunta, G.; Marcellino, L. Reconstruction of implicit curves and surfaces via rbf interpolation. Appl. Numer. Math. 2017, 116, 157-171. [CrossRef]

10. Lotfolah Hamedani, M.; Plimer, I.R.; Xu, C. Orebody modelling for exploration: The western mineralisation, broken hill, nsw. Nat. Resour. Res. 2012, 21, 325-345. [CrossRef]

11. Lajaunie, C.; Courrioux, G.; Manuel, L. Foliation fields and 3d cartography in geology: Principles of a method based on potential interpolation. Math. Geol. 1997, 29, 571-584. [CrossRef] 
12. Aug, C.; Chilès, J.-P.; Courrioux, G.; Lajaunie, C. 3d geological modelling and uncertainty: The potential-field method. In Geostatistics Banff 2004; Leuangthong, O., Deutsch, C.V., Eds.; Springer: Dordrecht, The Netherlands, 2005; pp. 145-154.

13. Frank, T.; Tertois, A.-L.; Mallet, J.-L. 3d-reconstruction of complex geological interfaces from irregularly distributed and noisy point data. Comput. Geosci. 2007, 33, 932-943. [CrossRef]

14. Cherpeau, N.; Caumon, G. Stochastic structural modelling in sparse data situations. Petrol. Geosci. 2015, 21, 233. [CrossRef]

15. Laurent, G.; Caumon, G.; Bouziat, A.; Jessell, M. A parametric method to model 3d displacements around faults with volumetric vector fields. Tectonophysics 2013, 590, 83-93. [CrossRef]

16. Laurent, G.; Ailleres, L.; Grose, L.; Caumon, G.; Jessell, M.; Armit, R. Implicit modeling of folds and overprinting deformation. Earth Planet. Sc. Lett. 2016, 456, 26-38. [CrossRef]

17. Guillen, A.; Calcagno, P.; Courrioux, G.; Joly, A.; Ledru, P. Geological modelling from field data and geological knowledge: Part ii. Modelling validation using gravity and magnetic data inversion. Phys. Earth Planet. Inter. 2008, 171, 158-169. [CrossRef]

18. Gois, J.P.; Trevisan, D.F.; Batagelo, H.C.; Macêdo, I. Generalized hermitian radial basis functions implicits from polygonal mesh constraints. Visual Comput. 2013, 29, 651-661. [CrossRef]

19. Zhong, D.-Y.; Wang, L.-G.; Bi, L. Implicit surface reconstruction based on generalized radial basis functions interpolant with distinct constraints. Appl. Math. Model. 2019, 71, 408-420. [CrossRef]

20. Hillier, M.J.; Schetselaar, E.M.; de Kemp, E.A.; Perron, G. Three-dimensional modelling of geological surfaces using generalized interpolation with radial basis functions. Math. Geosci. 2014, 46, 931-953. [CrossRef]

21. Hijazi, Y.; Knoll, A.; Schott, M.; Kensler, A.; Hansen, C. Csg operations of arbitrary primitives with interval arithmetic and real-time ray casting. In Scientific Visualization: Advanced Concepts; Dagstuhl Research Online Publication Server: Dagstuhl, Germany, 2010; pp. 78-89.

22. Goldfeather, J.; Monar, S.; Turk, G.; Fuchs, H. Near real-time csg rendering using tree normalization and geometric pruning. IEEE Comput. Graph. 1989, 9, 20-28. [CrossRef]

23. Wyvill, B.; Guy, A.; Galin, E. The blob tree-warping, blending and boolean operations in an implicit surface modeling system. Implicit Surf. 1998, 1-10. [CrossRef]

24. Wyvill, B.; Guy, A.; Galin, E. Extending the csg tree. Warping, blending and boolean operations in an implicit surface modeling system. Comput. Graph. Forum 1999, 18, 149-158. [CrossRef]

25. Hilton, A.; Stoddart, A.J.; Illingworth, J.; Windeatt, T. Implicit surface-based geometric fusion. Comput. Vis. Image Und. 1998, 69, 273-291. [CrossRef]

26. de Groot, E.; Wyvill, B.; van de Wetering, H. Locally restricted blending of blobtrees. Comput. Graph. 2009, 33, 690-697. [CrossRef]

27. Barthe, L.; Dodgson, N.A.; Sabin, M.A.; Wyvill, B.; Gaildrat, V. Two-dimensional potential fields for advanced implicit modeling operators. Comput. Graph. Forum 2003, 22, 23-33. [CrossRef]

28. Li, Q. Smooth piecewise polynomial blending operations for implicit shapes. Comput. Graph. Forum 2007, 26, 157-171. [CrossRef]

29. Fougerolle, Y.D.; Gribok, A.; Foufou, S.; Truchetet, F.; Abidi, M.A. Boolean operations with implicit and parametric representation of primitives using r-functions. IEEE Trans. Vis. Comput. Graph. 2005, 11, 529-539. [CrossRef]

30. Zhan, Q.; Chen, X. Boolean combinations of implicit functions for model clipping in computer-assisted surgical planning. PLoS ONE 2016, 11, e0145987. [CrossRef]

31. Macedo, I.; Gois, J.P.; Velho, L. Hermite radial basis functions implicits. Comput. Graph. Forum 2011, 30, 27-42. [CrossRef]

32. Faraj, N.; Thiery, J.-M.; Boubekeur, T. Multi-material adaptive volume remesher. Comput. Graph. 2016, 58, 150-160. [CrossRef]

33. Huang, Z.; Zou, M.; Carr, N.; Ju, T. Topology-controlled reconstruction of multi-labelled domains from cross-sections. ACM Trans. Graph. 2017, 36, 1-12. [CrossRef]

34. Newman, T.S.; Yi, H. A survey of the marching cubes algorithm. Comput. Graph. 2006, 30, 854-879. [CrossRef]

35. Schneider, P.; Eberly, D. Geometric Tools for Computer Graphics, 1st ed.; Elsevier: Amsterdam, The Netherlands, 2003 ; pp. 481-662.

36. Wold, S.; Esbensen, K.; Geladi, P. Principal component analysis. Chemometr. Intell. Lab. 1987, 2, 37-52. [CrossRef]

37. Zou, H.; Hastie, T.; Tibshirani, R. Sparse principal component analysis. J. Comput. Ggraph. Stat. 2006, 15, 265-286. [CrossRef]

38. Treece, G.M.; Prager, R.W.; Gee, A.H. Regularised marching tetrahedra: Improved iso-surface extraction. Comput. Graph. 1999, 23, 583-598. [CrossRef]

39. Ying, L. A kernel independent fast multipole algorithm for radial basis functions. J. Comput. Phys. 2006, 213, 451-457. [CrossRef]

40. Zhong, D.-Y.; Wang, L.-G.; Lin, B.; Jia, M.-T. Implicit modeling of complex orebody with constraints of geological rules. Trans. Nonferr. Metal. Soc. 2019, 29, 2392-2399. [CrossRef]

41. Guo, J.; Wu, L.; Zhou, W.; Li, C.; Li, F. Section-constrained local geological interface dynamic updating method based on the hrbf surface. J. Struct. Geol. 2018, 107, 64-72. [CrossRef] 